\title{
Cognitive Modeling of Socio-Economic Systems with Functional Consideration of the Time Factor
}

\author{
Aleksey Rogachev ${ }^{1,2, *}$, and Elena Melikhova ${ }^{3}$ \\ ${ }^{1}$ Volgograd State Technical University, Volgograd, Russian Federation \\ ${ }^{2}$ All-Russian research Institute of irrigated agriculture, Volgograd, Russia Federation \\ ${ }^{3}$ Volgograd State Agrarian University, Volgograd, Russian Federation
}

\begin{abstract}
The research analyzes scientific and methodological approaches for structural and parametric identification of fuzzy cognitive models based on time-dependent functional dependencies. In the process of constructing and identifying fuzzy cognitive models, the stages of structural identification with the definition of a set of concepts and unclear relationships over this set, parametric identification, which implements the transition to fuzzy identification with the definition of the intensity of influence between factors, are implemented. A specialized software system was developed for the purpose of computer support for modeling and studying the influence of the time factor set by changes in the strength of connections between concepts. A representation of each of the elements of the FCM adjacency matrix is proposed in the form of an additive expression controlled by the alpha parameter, which includes a constant component determined by the expert method and a control function that depends on real or dimensionless model time. To correct the elements of the FCM adjacency matrix, it is proposed to use a 3-parameter parabolic or modified 4-parameter exponential dependence as a function that depends on real or dimensionless model time. The developed modified method for solving the problem of time factor accounting using functional dependencies for the strength of mutual influence of concepts provides for expanding the capabilities of fuzzy cognitive models by taking into account the time factor.
\end{abstract}

\section{Introduction}

Identifying trends in the development of socio-economic systems, such as food security (FS), is a scientific problem that is important for improving methods and approaches to managing complex systems $[1,11]$. The use of the cognitive approach based on the construction, structural-parametric identification, and research of fuzzy cognitive maps (FCM) is limited by methodological difficulties of modeling in real time [2, 7, 12]. Based on a systematic approach, a review of foreign and domestic publications has revealed

\footnotetext{
*Corresponding author: rafr@mail.ru
} 
promising areas of time series data Mining, which combines statistical, fuzzy-multiple, hybrid neural network and cognitive technologies for analyzing multidimensional time series $[2,12,15]$. Cognitive methods allow us to analyze indistinctly defined data and predict not only the direction of changes in the MVR, but also to generate brief explanations of the evolution of such series in a linguistic form [12].

Cognitive modeling is the study of the structure, functioning, and development of the system under study by analyzing its cognitive map [4-6]. The classical cognitive model is an effective tool for evaluating the development of a system, but it does not make it possible to obtain quantitative characteristics of the evolution of the system under study in real time. The cognitive model can provide search and development of effective solutions for managing the system, as well as conduct scenario modeling that allows identifying risks and developing strategies to reduce them [3]. Scenario modeling allows you to predict the state of the simulated system under various control actions and search for alternative solutions that bring the system to the target state.

The main tasks that can be effectively solved using cognitive modeling can be divided into two groups [4]: tasks of structural and target analysis; tasks of dynamic analysis. When formulating and solving the problem of dynamic analysis, both the direct problem ("what will happen if...") and the inverse problem ("how to achieve...") can be solved. The mathematical apparatus underlying the methods of analyzing cognitive models in the form of fuzzy cognitive maps is fuzzy logic. A cognitive map with an indistinctly defined interaction between concepts is called a fuzzy cognitive map (FCM). The cognitive model can take into account that the intensity of mutual influences between concepts can be not only constant, but also variable $[2,4,7]$.

Expert methods used for parametric identification of FCM can be direct and indirect. Indirect methods are based on splitting the problem of determining the weights of the interaction of factors into simpler subtasks (the Saati Method, the Jager level method, the Churchman-Akoff method, etc.) [5,9]. If there is statistical information about the values of factors, it can be used to identify the weight coefficients of the relationship between such concepts instead of expert evaluation [10]. However, there are a number of methodological problems associated with the identification of fuzzy cognitive models based on statistical data [5].

\section{Materials and methods}

The research uses fundamental methods of system analysis - systems theory, cognitive analysis, algorithm theory, and multidimensional time series theory.

In the process of constructing and identifying fuzzy cognitive models, there are stages of structural identification (definition of a set of concepts $\mathrm{E}$ and a clear relation $\mathrm{W}$ over this set); parametric identification (transition from a clear relation $\mathrm{W}$ to a fuzzy one with determination of the intensity of influence between factors.

The result of formalization of the system in the form of a cause-and-effect network has the form:

$$
G=<E, W, t>
$$

where $E=(\mathrm{e} 1, \mathrm{e} 2, \ldots, \mathrm{ek})$-is the set of factors of the simulated system;

$W\left(w_{i j}\right)$ - is a binary relation on the set $E$ that defines the set of cause-and-effect relationships between its elements, $-1 \leq w_{i j} \leq 1$;

$t$-dimensionless or real time.

Graphically, FCM can be represented as a weighted oriented graph, whose points correspond to the elements of the set, and whose du-GI correspond to the non-zero elements of the relation $\mathrm{W}$. The relation $\mathrm{W}$ can be represented as a matrix of dimension $n \times n$ (where $\mathrm{n}$ is the number of factors), which can be considered as the adjacency matrix of the graph. 
To describe the FS support system, based on domain analysis, the FCM was constructed in the form of a weighted directed graph, the structure and parameters of which were set by the adjacency matrix $\mathrm{A}\left(\mathrm{a}_{\mathrm{ij}}\right)[8,14]$.

The adjacency matrix that models the interaction of concepts has the form

$$
\boldsymbol{A}=\left\|a_{i j}(t)\right\|
$$

where $\mathrm{A}$ - is the adjacency matrix of the modeling FCM,

$a_{i j}$ - is an element of the matrix A, and $t$ is dimensionless or real time.

The preliminary stage of cognitive modeling, carried out in order to justify the composition of the key concepts of the simulated system, was provided using the Strategist computer system developed at the Volgograd state technical University [13]. The system has a user-friendly interface that provides interactive construction, visualization, and research of a fuzzy cognitive model.

For the purpose of computer support for modeling and studying the influence of the time factor set by changes in the strength of connections between con-cepts, a special software system was developed, the interface of which is presented below.

\section{Results and discussion}

The authors propose to set the values of the adjacency matrix elements that characterize the mutual influence of FCM concepts in the form of two components:

- a constant value set by experts,

- a time-dependent variable component, also set by experts, from which you can write.

$$
a_{i j}=\alpha \cdot w_{i j, e}+\varphi(t) \cdot(1-\alpha)
$$

where $\alpha$ - is a dimensionless parameter that takes into account the share of influence of the time component on the element of the matrix $A$;

$w_{\mathrm{ij}, \mathrm{e}}$ - expert (fuzzy) coefficient of influence of concept $i$ on concept $j$;

$\varphi(t)$ - some functional dependence.

The choice of the type of functional dependence $\varphi(t)$ is determined by a priori views of experts about the nature of changes in the mutual influence of concepts. Then, for a sufficiently universal parabolic dependence (3), we can write an expression for the elements of the matrix $A$

$$
a_{i j}=\alpha \cdot w_{i j, e}+(1-\alpha)\left(A_{i j} \cdot t^{2}+B_{i j} \cdot t+C_{i j}\right)
$$

where $a_{i j}$ - is an element of the matrix A;

$t-$ is the dimensionless time.

$$
\varphi(t)=A_{i j} \cdot t^{2}+B_{i j} \cdot t+C_{i j}
$$

where $\mathrm{A}, \mathrm{B}$, and $\mathrm{C}$ are the parameters of a parabolic function that defines the change in time of the coefficients of influence of concepts.

Note that the parameters of the parabolic function (5) are estimated from the analysis of the dynamics of statistical data for a known period. At the same time, the dependence (5) can also be used for matching the model time with the real one.

The value of parameters A, B, and C of the parabolic dependence (5) is chosen from a priori considerations, taking into account expert judgments. For the case of symmetric arrangement of branches of a quadratic parabola (5), we have (Fig. 1-a). 


$$
B=0 ; \varphi(0)=C ; A<0 ; \varphi(\text { tend })=0,
$$

where tend is the real or dimensionless time of completion of the steady-state simulation process.

The value of parameter A can be determined from conditions (6) by solving the equation (7)

when $t=$ tend.

$$
A_{i j} \cdot t^{2}+C_{i j}=0
$$

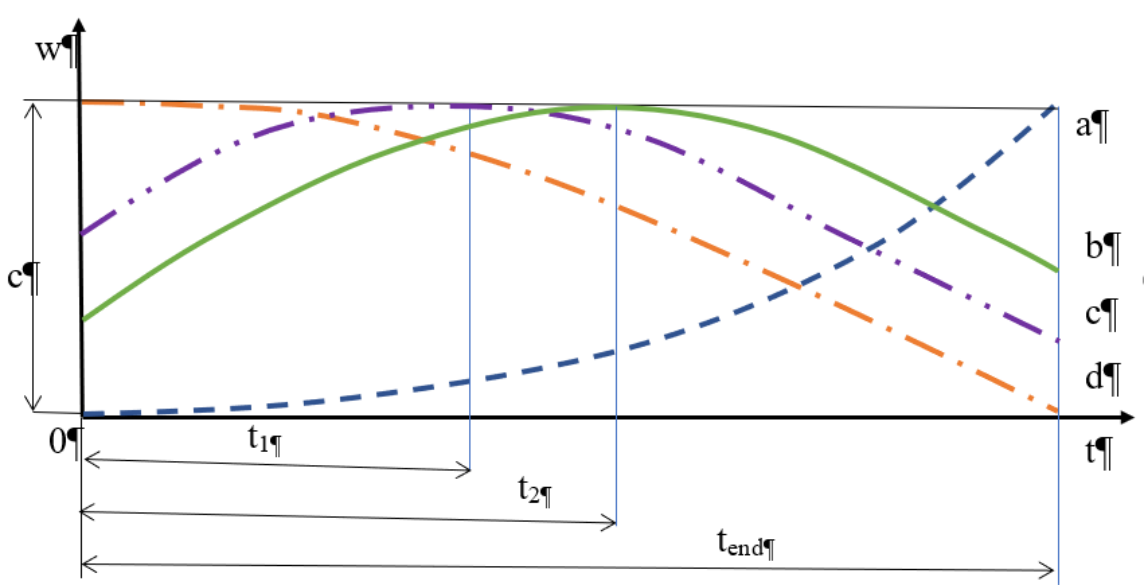

Fig. 1. Forms of functional dependencies of adjacency matrix elements on time

If it is necessary to have as a function $\varphi(t)$ monotone dependencies (increasing or decreasing), only one of the branches of the quadratic parabola can be used to smoothly change the values of the elements a_ij of the matrix A.

In the case when $A \neq 0$ and $B \neq 0$, it is possible to obtain a non-monotonic dependence (Fig. 1-b, 1-c) for the function $\varphi(t)$.

Addition to the parabolic one, the dependence $\varphi(t)$ can also take a more complex form, for example, in the form of a modified 4-parameter exponential function

$$
Y_{t}=\beta_{0}+\frac{\beta_{1} \times\left(t-\beta_{3}\right)}{1+\exp \left(-\beta_{2} \times\left(t-\beta_{3}\right)\right)},
$$

where $\beta i$ - are parameters; $t$ - is real or dimensionless time.

Since the computer system "Strategist" does not allow you to set functional dependencies of any kind, it was necessary to develop a specialized software system that provides flexible configuration of such functions using interactively entered parameters for each combination of concepts $i$ and $j$.

The developed system allows you to set the values of elements of the adjacency matrix in the form of functional dependencies on time, which ensures that it is taken into account in cognitive modeling.

The cognitive map on the example of modeling food security through includes 11 concepts. Concepts define the basic factors of production, consumption, food stocks and imports, as well as economic infrastructure and industrial-technological interactions. The time-dependent components of the adjacency matrix elements for modeling the food security system were set in the form of monotonically decreasing values realized by the right branches of quadratic parabolas (Fig. 1-d). The exception was the dependence of the influence of the "Ecology" factor on the integral level FS, the significance of which was assumed to be increasing (fig. 1-a). 


\section{Conclusions}

The study of the problems and possibilities of taking into account the time factor in fuzzy cognitive modeling, using the example of FS modeling, allowed us to obtain the following conclusions:

1. The considered methods of constructing and parametric identification of fuzzy cognitive models, including existing expert and statistical methods, revealed the need to develop or modify methods for solving this problem in order to expand the functional capabilities of fuzzy cognitive models.

2. we Propose a representation of each of the elements of the adjacency matrix FCM in the form of an additive expression controlled by the alpha parameter, including a constant component determined by the expert method and a function that depends on real or dimensionless model time.

3. to correct the elements of the adjacency matrix FCM, it is proposed to use a 3parameter parabolic or modified 4-parameter exponential dependence as a function that depends on real or dimensionless model time.

\section{Acknowledgements}

The article was prepared with the financial support of RFBR under the project 19-07-01132.

\section{References}

1. I. M. Azhmukhamedov, Proble. of Inf. Secur. Comp. syst., 1 (2013)

2. V. Borisov, V. Luferov, Syst. of Contr., Communic. and Secur., 2 (2020)

3. A.N. Averkin, S.A. Yarushev, V.Yu. Pavlov, Programmnye produkty i sistemy (Software \& Systems), 30(4) (2017)

4. R. Isaev, A. Podvesovskii, CEUR Workshop Proceedings of the International Conference on Information Technology and Nanotechnology, 2, 212 (2018)

5. A. Podvesovskii, R. Isaev, International Journal of Open Information Technologies, 7(6) (2019)

6. S.V. Podgorskaya, A.G. Podvesovskii, R.A. Isaev, N.I. Antonova, Business Informatics, 13(3) (2019)

7. N. B. Paklin, Artificial intelligence, 4 (2003)

8. A. Rogachev, E. Antamoshkina, IOP Conf. Ser.: Earth and Envi. Sci., 403 (2019)

9. D. V. Erokhin, D. G. Lagerev, E. A. Laricheva, A. G. Podvesovskii, Strategic Enterprise Innovation Managemnet: Monograph (2010)

10. A. F. Rogachev, E. V. Melikhova, Problems of statistical estimation of cognitive map characteristics on the basis of correlation analysis Proc. of the Int. Conf. "Phys.-Math. Sci.: Theory and Practice" (2014)

11. A. Zagranovskaya, Bulletin of Plekhanov Russian University of Economics, 4(100) (2018)

12. N. G. Yarushkina, T. V. Afanasyeva, I. G. Perfileva, Intellectual analysis of time series: a textbook (2010)

13. S. Fomenkov, D. Korobkin, V. Kamaev, Theoretical foundations of system modeling: textbook. manual (2016) 
14. Food Security Doctrine of the Russian Federation the Presidential Decree from 30.01.2010, Rossiyskaya Gazeta 5100 (2010)

15. K. Tokarev, IOP Conf. Ser.: Earth and Env. Sci., 421(4) (2020) 